has been developed to introduce patients with an early diagnosis of MND to the hospice. This may help patients access hospice services sooner.

\section{0 'PALLIATIVE CRITICAL CARE': AN IMPORTANT AND TRANSFORMATIVE COLLABORATION BETWEEN PALLIATIVE CARE AND INTENSIVE CARE SERVICES AT A LARGE TEACHING HOSPITAL}

J Westwood, H Crispin, K Haynes, E Gowling, R Chambers, C Davis. University Hospital Southampton NHS FT

\subsection{6/spcare-2020-PCC.180}

Introduction In 2013, the Hospital Specialist Palliative Care (HSPCT) and General Intensive Care Unit (GICU) Teams began a quality improvement initiative in response to the palliative care needs experienced by GICU patients. 'Palliative Critical Care' now involves seamless inter-disciplinary working on an almost daily basis, supported by one session each per week of dedicated Intensivist and Palliative Medicine Consultant time. Here, we detail the impact of this collaboration on HSPCT activity and patient outcomes.

Methods Retrospective review of HSPCT records between April 2013 and March 2019.

Results Over the last 5 years the HSPCT has received 588 referrals from adult intensive care services (General, Cardiac and Neurological). From a baseline of 6 referrals in 2013/14 we now receive over 130 new referrals per year. Over this time our caseload has evolved; $79 \%$ of referrals for nonmalignant conditions in 2018/19, compared with 59\% in $2014 / 15$. In both years the in-hospital mortality rate of patients seen by the HSPCT was approximately 50\%.

Key areas of impact include pain control, symptom control, family support in $66 \%, 81 \%$, and $86 \%$ of interactions, respectively. Patients are given psychological support in $48 \%$ of interactions. The HSPCT also provide valued support to ICU staff in $32 \%$ of interactions and discharge advice in $19 \%$.

HSPCT involvement now routinely continues throughout the patient's hospital stay. The average number of contacts per patient has increased from 4.3 to 7.5 (2014/15 vs 2018/19, respectively), each contact lasting 30-40 minutes. Thus, the average total duration of HSPCT time per patient has increased from 139 minutes (2014/15) to 287 minutes (208/ 19).

Conclusion This Quality Improvement Initiative has enabled a flourishing collaboration between our HSPCT and GICU teams resulting in a sustained increase in referral numbers from all adult ICU areas with more time dedicated to support each patient in a continuously evolving cohort.

\section{SETTING UP A HOSPICE BASED RENAL SUPPORTIVE CARE SERVICE: WHAT WE HAVE LEARNED OVER THE LAST 3 YEARS}

Jane Whitehurst, Sue Goodall, Ellie Hayter, Kevin Blackett. St Barnabas House

10.1136/spcare-2020-PCC.181

Background St Barnabas House Renal Supportive Care (RSC) Service for patients with advanced renal disease $(\mathrm{CKD}<15)$ launched in 2016. Model: RSC clinical nurse specialist (CNS) 32 hrs per week; palliative care consultant support within existing community sessions; hospice multidisciplinary team input. Intervention: domiciliary visits, hospice-based outpatient clinics, joint reviews in nurse-led hospital renal clinic, attendance at hospital renal service (HRS) complex patients MDM; symptom control including fluid management; advance care planning (ACP); decision making support regarding treatment modality; Psychosocial support; liaison with community services and HRS.

Methods Service evaluation using system 1 data for patients on RSC caseload April 2016 to August 2019. Identifying successes, challenges and future opportunities.

Results Strengths: Increased access from \&lt; $1 \%$ to $3 \%$ of hospice annual referrals, $49 \%$ of patients accessed other hospice services; $87 \%$ of patients who died, died in a community setting; good feedback from patients; collaborative working with HRS.

Challenges Initial referral criteria included an eGFR $<15$ and patient's choice to pursue conservative management, both have limited value in predicting prognosis or specialist palliative care needs; Patients undergoing dialysis are underrepresented (17\%); the traditional model of hospice care acceptance onto caseload from referral until death, is less relevant in this group; unclear monitoring/prescribing responsibilities for renal specific symptoms - diuretics, anaemia and electrolytes; lack of a supportive care register or clinical lead for HRS.

Opportunities Modification of referral criteria - needs rather than diagnosis based; modification of service model to deliver care in episodes; further collaboration with HRS - shared symptom scale, clearer shared care responsibilities.

Conclusions The RSC service at $\mathrm{StBH}$ has increased access to specialist palliative care for people with advanced renal disease. However the described challenges could negatively impact a sustainable and safe service as referral numbers increase. We have identified and are implementing solutions working collaboratively with the HRS.

\section{EVALUATION OF SPECIALIST PALLIATIVE CARE SERVICES TO THE GYPSY AND TRAVELLER POPULATION IN THE LEEDS AREA}

Hannah Wilson, Marianne Ralph, Melanie Clarkson. Wheatfields Hospice, Sheffield Hallam University

\subsection{6/spcare-2020-PCC.182}

Background The health of Gypsy and Traveller communities is significantly worse than the health of other minority ethnicities, experiencing premature mortality and greater risk of illness. The Leeds Community Health Needs Assessment in 2013 identified poor access to health services, especially within primary care. Recent evidence has highlighted that end of life care needs of Travellers and Gypsies must be recognised, understood, and supported by services both nationally and locally. In order to achieve this we needed to understand our current palliative care service provision and the barriers from both service users and professionals to achieving wider access to palliative services in this community

Methods A service evaluation was undertaken focussing on the care provided within an area served by Sue Ryder 